\title{
A Strategic Approach to Transformational Design
}

\author{
Jürgen Bohn \& Wil Janssen * \\ University of Oldenburg $\star \star$
}

\begin{abstract}
Designing parallel systems in a correct way is difficult. Transformational design of systems guarantees correctness by the correctness of the transformations, but is often tedious and complicated. We discuss different transformation strategies to guide the designer from the initial specification to different implementations, tailored to different architectures. Strategies give rise to simpler transformation rules, point the way in the design trajectory, and allow for the reuse of proofs and transformation steps when deriving optimizations and variants of algorithms.
\end{abstract}

\section{Introduction}

Correct design of parallel and distributed algorithms and protocols is a difficult task. Such algorithms are often the result of numerous design decisions and architectural optimizations, and are therefore very hard to prove correct. When designing such systems in a transformational way from a specification, correctness with respect to the initial specification is obtained for free due to the correctness of the transformation steps.

Although transformational design in principle solves the correctness issue, it is very hard to apply to larger systems. In developing systems transformationally one often has an abundance of choices between different transformation rules. It is difficult to decide which way to go, and decisions taken at an early stage can have severe influences in the rest of the design trajectory. Thus it is very easy to "get lost" in the design process. Moreover, transformation rules are often rather complicated as they are designed for a general setting. Also they often concern only small steps, leading to a very long design trajectory. Finally, having derived one algorithm, it would be desirable to be able to reuse the proofs and steps used in other derivations of similar systems.

In such a transformational design process the design engineer needs guidance in order to keep him or her from getting lost. We propose the use of strategies for this purpose. A strategy describes what type of transformation should be applied at what stage of the design process. One can think of many different strategies for developing parallel systems. A first strategy is called the layered strategy. In this strategy the first steps in the design are restricted to sequential or layered program development. After having obtained a sufficient level of detail the resulting design is then parallelized to

* This work was partially supported by the Deutsche Forschungsgemeinschaft under grant No. Ol 98/1-1.

** Fachbereich Informatik, P.O. Box 2503, D-26111 Oldenburg, Germany. E-mail: \{Juergen.Bohn, Wil.Janssen\} Qinformatik.uni-oldenburg.de 
meet the desired architecture, and finally implemented. Examples of this approach are the work by Elrad and Francez [8], by Chou and Gafni [7], Stomp [23], and by Janssen, Poel and Zwiers [14, 13].

A second strategy is the parallel strategy, where at the initial stages one is not concerned about architectural issues but simply views the system as a set of continuously executing actions. In the later stages one then introduces architectural decisions as optimizations, leading to an implementable algorithm. The most prominent example of this approach is the UNITY approach as developed by Chandy and Misra [6]. The work by Back and Sere on action systems [1,2] also takes this viewpoint.

A third strategy is called the architectural strategy, where one aims at a specific architecture already at the very initial design stages, thus giving a more direct design trajectory. This approach has been employed by Olderog et al. in the ProCoS project $[18,19,20]$.

Having recognized a certain strategy one can develop specialized rules that are tailored for certain design stages and therefore can be much more restricted and thus simpler. Due to the simplicity, such strategies can be amenable for tool support. Also, if a certain strategy applies nicely to a class of algorithms, the derivations of the different algorithms in that class often share large parts and have similar steps at similar stages. Thus strategies encourage reuse of proofs.

In this paper we discuss the three strategies mentioned above, and use them to derive different implementations of atomic commit protocols for different architectures, such as Centralized Two-Phase Commit, Linear Two-Phase Commit, and Decentralized Two-Phase Commit (see Bernstein, Hadzilacos and Goodman [3] for a discussion of these protocols). Atomic commit protocols are used in distributed database systems to guarantee consistent termination of transactions that are executed at different sites in a database network.

As a vehicle for transformational design we use the mixed terms approach as has been developed in the ProCoS project [19]. There a specification language SL has been developed that allows to specify reactive behavior by means of regular expressions, as well as state transitions in $\mathrm{Z}$ style. Such specifications are also part of a mixed terms language MIX which allows to transform specifications up to a point where they correspond to CSP style language constructs [12] and can be implemented in OCCAM. As SL allows to specify both aspects, reactive behavior and state transitions, it is very well suited to handle the different aspects of the different strategies. Besides that, the transformational theory for SL and MIX is well developed [5, 20, 19].

For a detailed account of the different transformations and a discussion of the derivations of other architectures we refer to [4].

\section{Strategies and Their Ingredients}

In the introduction we mentioned three different strategies to develop parallel and distributed systems transformationally. In this section we discuss these strategies in more detail, and list what types of transformations are needed for such strategies to work out nicely. Different strategies also have different application areas they are most suited for. 
By no means we want to claim that these three are the only strategies one can think of, but they are three well-known approaches to program development and therefore we restrict ourselves to these three.

\subsection{The layered approach}

The design and analysis of sequential programs is inherently much simpler than of parallel programs. One does not have to take interference of other components into account, and no deadlock can occur due to synchronization. For parallel and distributed systems it has been observed that they often can be analyzed in a sequential or layered fashion as well. This is already obvious from the terminology used: one talks about "two-phase" or "three-phase" commit protocols, termination "phases" or connection "phases" in communication protocols etcetera. Such "phases" are also sometimes called layers, which should not be mistaken for the term "layers" as used in the OSI reference model.

Elrad and Francez [8] used this observation to formulate the so-called communication closed layers principle that states that under certain circumstances a layered and a parallel program are equivalent. This principle has later been used in a transformational setting by, amongst others, Stomp [23] and by Janssen, Poel and Zwiers [14, 13]. Similar ideas of independence also play a crucial role in parallelization of loops in conventional programs (see, for example, Lengauer [16]). Also in the work by Back and Sere [2] one uses transformations to go from initial a sequentially structured solution to parallel implementations. Their work is formulated using action systems.

Based on these ideas we can formulate the following layered strategy.

(L1) Given a specification or initial design (that is correct by definition), repeatedly decompose the specification in sequence of (abstract) actions, using standard techniques as known for sequential program development.

(L2) If one arrives at a sufficient level of detail, for example at an implementable level, the resulting algorithm is analyzed for possible parallelization: Independence of actions indicates potential parallelism, where independence is determined on syntactic grounds or on semantic grounds in the most general case.

(L3) Finally, the resulting system consisting of the parallel composition of sequential components is transformed into a real, fine-tuned implementation.

Note that in this strategy there is not explicit reasoning on parallelism, only independence analysis is needed. For this strategy to be applicable one requires easy laws for sequential decomposition and independence analysis. It has been shown in the work mentioned above that this strategy is applicable to a large class of systems, although it is by no means a complete strategy.

\subsection{The parallel approach}

A similar idea of avoiding architectural bias also is the basis of the parallel approach. In this strategy, as for example employed in the work on UNITY [6] by Chandy and Misra, 
or by Back and Sere using action systems [2], one first derives an unstructured but complete implementation, after which one defines a mapping of actions and variables to processes satisfying the constraints induced by the desired architecture.

This approach goes through the following phases.

(P1) Given a certain specification of the problem, develop a set of actions as basic ingredients of the eventual implementation.

(P2) Prove that all runs consisting of the actions in the set under a weakly fair scheduling policy lead to a stable state satisfying the desired end state.

(P3) Define a mapping of variables to processors, and define sequences of actions from the initial action set that are weakly fair for each processor, satisfying constraints induced by the communication structure between the processors.

(P4) Rewrite the initial unstructured solution under the mappings defined in a previous step to an actual implementation.

The most difficult part in this is proving that the actions indeed lead to the desired stable state. For this one can use, for example, the UNITY logic as introduced in [6]. Also, depending on the desired architecture, the definition of the mappings can require encoding of channels or buffers, which is not always as natural as one would hope for. In general however, the application area of this approach is not limited to a specific class of algorithms.

\subsection{The architectural approach}

In the layered and parallel strategies the architectural decisions are postponed to as late as possible, allowing different implementations. However, often one has a very clear idea of the architecture the system should function in, for example a fully connected network with $n$ nodes, or a two processor shared memory architecture. Incorporating such information at a very early stage might allow to simplify intermediate specifications or systems to a large extent.

This approach has been taken in the ProCoS project to design synchronously operating parallel CSP style systems $[18,5]$. The architectural approach goes through the following four phases:

(A1) Given an initial specification, one adds to this specification the synchronization skeleton of the system one is aiming at, so for each process the sequence of synchronizing actions is specified.

(A2) The state space of each process is extended with the required local variables.

(A3) For every action, the corresponding state transformation part of the initial specification is moved to it, making the initial specification redundant.

(A4) Analogously as for the layered approach, the resulting system is transformed into an implementation. 
Addition of synchronization skeletons and moving (parts of) state transformations are two important ingredients here. Both transformations can be performed very nicely in the mixed terms language MIX, as is discussed in section 4. Moreover, this approach is not bound to a certain class of algorithms, although due to its directness, reuse and structuring of proofs seems to be more difficult.

\section{Atomic Commit Protocols}

Distributed systems consist of a collection of sites that are connected by means of some network. In a distributed database system an important concept is maintaining consistency of the different sites. Each site has a local database system that maintains a part of the distributed database. A distributed transaction consists of a sequence of reads and writes to database items. We assume every transaction $T$ has a "home site" where it originated. Termination of a transaction $T$ is intricate, as it concerns all sites that participated in $T$. Simply sending a message to a site stating that it should commit is not sufficient: it is possible that - for example due to failures of storage media or volatile memory - a site cannot store the changes to the database, and therefore cannot commit. The fact that a single site is not able to commit should result in aborting the transaction, which implies aborting at all sites involved in order to keep the distributed database consistent.

An algorithm that guarantees consistent termination of distributed transactions is called an Atomic Commit Protocol (ACP). We assume the transaction involves a coordinator process $C$ at the home site, and a set of participating processes $P$ for all sites that were accessed. Every participating process has one vote: YES or NO, and every process can reach one of two decisions: COMMIT or ABORT. An ACP should guarantee that every process votes, and eventually makes a decision, where the decision should be COMMIT iff every process voted YES, and ABORT otherwise. (See [3] for details.)

A well-known example of an ACP is the Two-Phase Commit Protocol (TPC), which operationally behaves as follows. After the process at the home site (the coordinator) has received a signal it requests all participating processes to send their votes. When it has gathered all votes it decides according to these votes and sends the decision back to all participants, which decide accordingly. This protocol is formulated for a system with a coordinator that can communicate with all participants, which is called centralized TPC. There exist also solutions for other network architectures. For example, if we have a fully connected network, all processes can send their votes to all other processes, after which every process can make the decision itself. This is called decentralized TPC.

\section{Specifications and Implementations}

The ESPRIT Basic Research Actions ProCoS I and II investigate the trustworthy design of embedded systems over the whole development process ranging from requirements capture over specification and programming level down to transputer machine code [9]. As part of this project a refinement calculus for communicating systems was developed in order to provide a constructive and mathematically sound way for bridging the gap 
between specifications and programs [17, 20]. From ProCoS we take the specification language SL [20] for communicating systems. SL combines regular expressions, process algebra and action systems.

Simple specifications in SL consists of an interface $\triangle$, a set $T A$ of trace assertions, a set $l V$ of local variables and a set $C A$ of communication assertions, written as

$$
\text { system } \triangle T A I V C A \text { end. }
$$

The interface provides a static view of the specified system. Components from $\Delta$ are directed channels (input or output) and global typed variables (read or write).

Trace assertions have the form "trace on $\alpha$ in $r e$," where the alphabet $\alpha$ is a set of channels from $\Delta$ and $r e$ is a regular expression over $\alpha$. They define sequencing constraints on the global communication order using regular expressions over channel names, that is any sequence of communications projected to $\alpha$ has to be a word in the language defined by re. Using more than one trace assertion allows to focus on different aspects concerning different channel sets separately.

The state part of a specification encompasses local variable declarations and communication assertions. In the style of Z [21] and TLA [15], the latter specify what the values are that are communicated and how the single communication actions will change the system state. Such communication assertions are of the form

$$
\text { com } a \text { read } \bar{x} \text { write } \bar{y} \text { when } w h(\bar{x}, \bar{y}) \text { then } t h\left(\bar{x}, \bar{y}, \bar{y}^{\prime}\right) \text {, }
$$

where $\bar{x}$ and $\bar{y}$ are lists of variables read and written respectively, and $w h$ is a predicate defining when action $a$ is enabled, and where th specifies the result of the action. As in, for example, $Z$ we use primed versions of variables to refer to their new values after the communication. We use the channel or action names prefixed by the symbol "@" as logical variables referring to the values communicated on these channels. Simple specifications may be enriched by local channels and state restrictions such as initializations and invariants.

As the target programming language we consider an OCCAM-like language PL, with basic processes SKIP, STOP, assignment, input and output communications, WHILE loops and compositional operators for sequences, alternatives, conditionals and concurrency.

To perform transformations from SL to PL we work in a "combined language" MIX which contains SL and PL as subsets. MIX consists of so-called mixed terms that may apply program operators to specifications. The transformational approach is based on a common semantic model for SL, PL and MIX. It is a trace-state-readiness model [20], which is well suited for describing all features such as synchronous communication, parallelism, nondeterminism and states.

Starting from a given SL specification Spec, a correct implementation Prog is derived by iterated application of transformation rules such that the specification notation is gradually replaced by PL constructs.

$$
\text { Spec } \equiv S_{1} \Leftarrow \cdots \notin S_{n} \equiv \operatorname{Prog}
$$

Typically a transition step from mixed term $S_{i}$ to $S_{i+1}$ is performed by replacing some specification expression $S$ in $S_{i}$ by a mixed term $T$ where the refinement $T \Rightarrow S$ or 
even $T \equiv S$ is guaranteed by a transformation rule. Then the overall implementation correctness follows from the transitivity of " $\Rightarrow$ " and the monotonicity of all operators with respect to " $\Rightarrow . "$

\subsection{Specifying Atomic Commit}

Let us now explain the concepts of SL by the starting specification of the ACP. The idea is that the protocol performs a state transformation that we model by a single action $A C P$. Thus the only trace assertion is

$$
\text { trace on } A C P \text { in } A C P \text {, }
$$

where $A C P$ is an local channel/action and the regular expression $A C P$ states that the protocol is executed once only.

To describe the state space of the system we use local variables as follows: The vote given by every process is stored in a variable vote $_{i}$ and the decision taken by every process in $\operatorname{dec}_{i}$. The fact that every process has a way of checking whether or not it can COMMIT if needed is modeled by a boolean flag stable $_{i}$ for each process $i$. Since this decision is not done by the protocol but by the environment stable $i_{i}$ is a read variable in the interface (for all $i=1 . n$ ). The state transformation is specified by a single communication assertion for $A C P$.

The result is the specification presented in figure 1. In particular it states that from

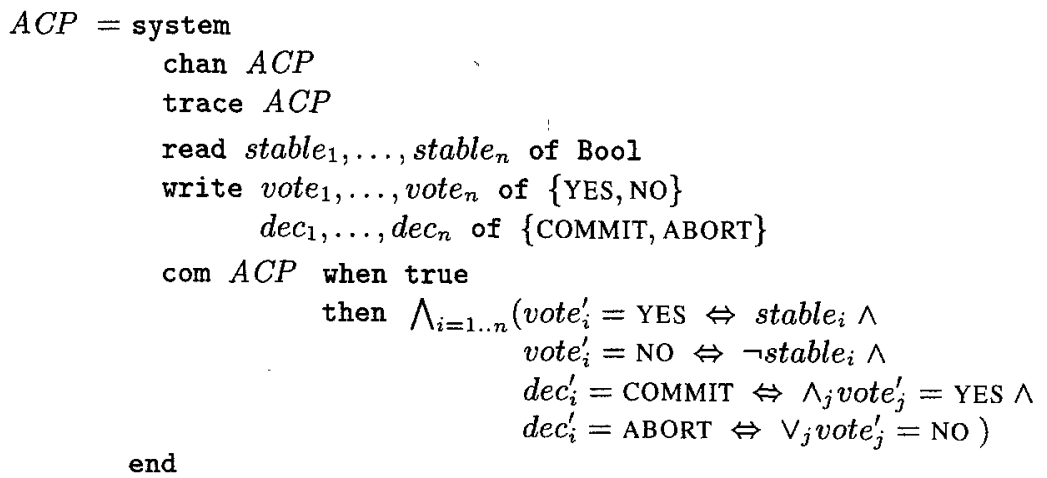

Fig. 1. Starting specification of $A C P$.

any initial state the protocol should terminate in a state where the decision for each process is COMMIT iff every process voted YES. Furthermore a process shall vote YES iff $s_{a} a b l e_{i}$ was true. Depending on the requirements of the environment the specification may terminate after an application or reach a stable state where it is ready for another protocol run. 


\subsection{A transformation rule}

In a transformational setting we use decomposition rules to refine systematically specifications like $A C P$. Decomposition means here that a more complex specification is replaced by a mixed term where some composition operator is applied to several simpler subsystems. As a typical example supporting this kind of refinements, figure 2 shows a

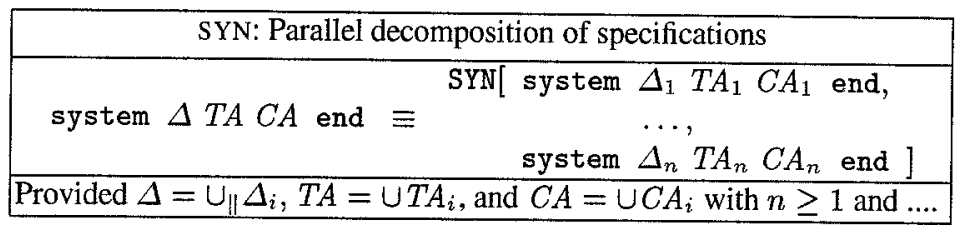

Fig. 2. Transformation rule SYN decomposition.

general transformation rule which introduces the synchronization operator SYN. Generally a side condition "provided ..." restricts the applicability of the transformation rule and describes how the new mixed term is derived by syntactic modifications from the given one.

At the end of the design process simple specifications are replaced in transformation steps by basic PL statements as for example input or output communications or assignments. Figure 3 shows appropriate equivalences of specification and programming constructs. Other simple specifications can be transformed into these patterns and are therefore automatically implementable.

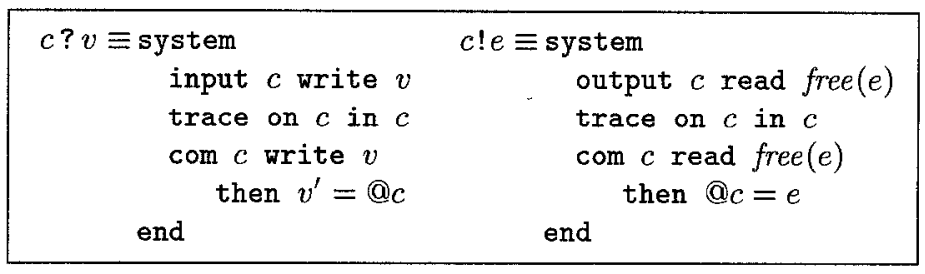

Fig. 3. Meaning of input and output communication statements in PL

\section{A Layered Derivation of TPC}

As a first derivation we will use the layered strategy to go from the initial specification $A C P$ to an implementation. The protocol we will aim at will be centralized TPC where a single coordinator process communicates with a set of participating processes.

The layered strategy uses two important ingredients: sequential decomposition and independence analysis. We first define specialized transformation rules to do so. 


\subsection{Decomposition and independence rules}

The general idea for sequential decomposition is rather simple. Assume we have a complicated action $a$. If we can split $a$ into two actions $a_{1}$ and $a_{2}$ such that the combined effect of executing $a_{1}$ and $a_{2}$ after one another is equivalent to $a$, then we would like to replace $a$ by the sequence $a_{1} . a_{2}$. The general rule to do so is rather complicated: one has to ensure atomicity of $a_{1} . a_{2}$ and also that no new actions become enabled by the extra intermediate state between $a_{1}$ and $a_{2}$. (See [20], $\mathbf{T} 5.19$, page 103.) In a sequential setting atomicity however is immediate, and no other actions can become enable as their order is fully specified.

The notion of "combined effect" is formalized by a contraction function contr that maps non-empty sequences of (local) actions to a single (local) action with the combined effect. Roughly speaking this amount to joining read and write lists of actions and combining the enable and effect predicate to a single enable and effect predicate. For the full definition we refer to [4]. All these simplifying circumstances lead to the rule given in figure 4, where $\bar{v}$ is a (possibly empty) set of local variables to store intermediate results, and $a \backslash \bar{v}$ is the action resulting from hiding the variables $\bar{v}$ from $a$. Moreover $t(c)$ denotes a trace expression where $c$ occurs as a subexpression in $t$.

\begin{tabular}{|c|c|}
\hline \multicolumn{2}{|c|}{ SSD: Simple sequential decomposition } \\
\hline $\begin{array}{l}\text { system } \triangle C A l V l C \\
\text { chan } c \text { trace } t(c) \\
\text { com } c \text { write } \bar{w} \text { read } \bar{r} \equiv \\
\text { when } w h \text { then } t h \\
\text { end }\end{array}$ & $\begin{array}{l}\text { system } \triangle C A l V l C \\
\quad \text { var } \bar{v} \\
\text { chan } c_{1}, \ldots, c_{n} \text { trace } t\left(c_{1}, \cdots, c_{n}\right) \\
\text { com } c_{i} \text { write } \bar{w}_{i} \text { read } \bar{r}_{i} \\
\text { when } w h_{i} \text { then } t h_{i} \\
\text { end }\end{array}$ \\
\hline Provided $c, c_{1}, c_{2}, \ldots$ & $\begin{array}{l}\notin \Delta \cup l C, \bar{v} \cap(l V \cup \Delta)=\emptyset, \\
\text { write } \bar{w} \text { read } \bar{r} \text { when } w h \text { then }\end{array}$ \\
\hline
\end{tabular}

Fig. 4. A rule for sequential decomposition

To derive parallel programs more is needed than sequential decomposition. Independence analysis and rules to introduce parallel components are needed. The latter was already introduced in section 4 . For this to apply we have to rewrite the single trace that is the result of the sequential decomposition into a set of (synchronizing) traces.

The simplest notion of independence is syntactic independence. Two actions $a$ and $b$ are syntactically independent, denoted by $a \not \alpha \rightarrow b$, iff every variable they share is only read by them and not written. This can be determined from the communication assertions as they specify the lists of variables read and written. A more general notion of independence can be defined if we take states into account. For example, two actions both increasing $x$ by one are independent in the sense that in what ever order they are performed, the overall effect remains the same. This however will not be used in this paper (see [4] for an application thereof). We say that $a$ and $b$ are dependent, denoted by $a \leftrightarrow s b$, iff they are not independent. Note that both dependence and independence are 
symmetric relations. A rule for parallelizing independent actions is given in figure 5. If

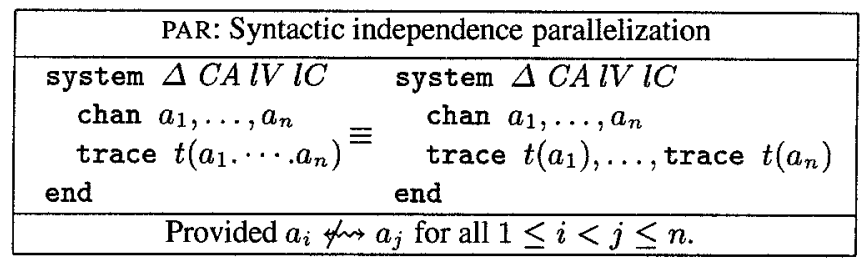

Fig. 5. A rule for parallelizing independent actions

besides independence also the side conditions for write accesses to shared variables are fulfilled and so on (as required for SYN decomposition) we can indeed replace the above specification by the synchronization of the sub specifications. In the case of other interfering trace assertions this last step cannot be taken as then no suitable decomposition of the different parts exists.

\subsection{The first decomposition steps}

We now have the ingredients for the derivation. As a first derivation step we decide to split the single action $A C P$ into a sequence of two abstract phases. The idea is that after the first phase the decision to be taken by the protocol should be known. To store this decision we introduce a local variable $d e c$. The result is $T P C_{1}$ in figure 6.

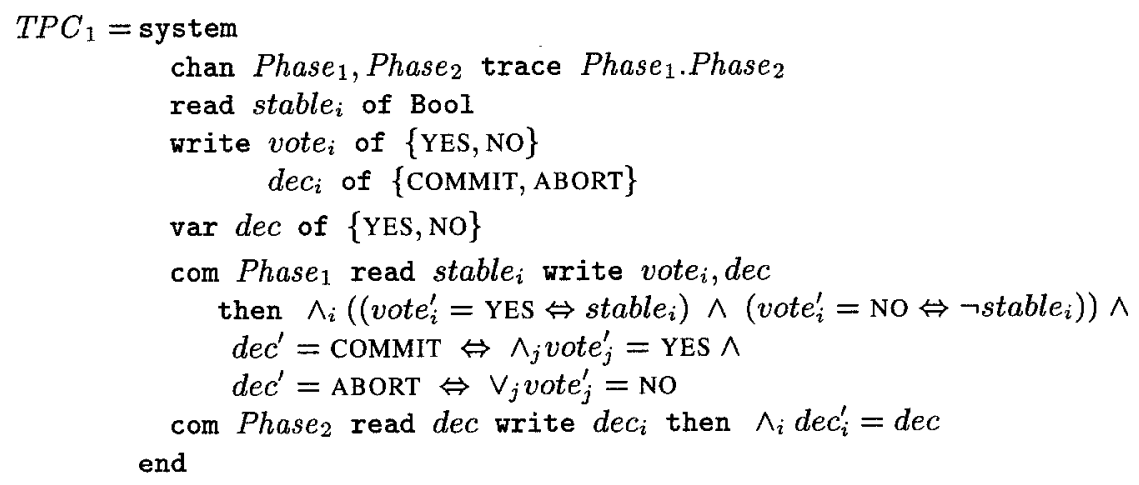

Fig. 6. The specification after the first decomposition step

The correctness of this step follows from the fact that the effect of $P_{h a s e}$. Phase $_{2}$ is the same as that of $A C P$. We now take a closer look at the first phase. There are 
several ways to split this action again. We split it into a voting action and an action where the decision is taken based on the votes.

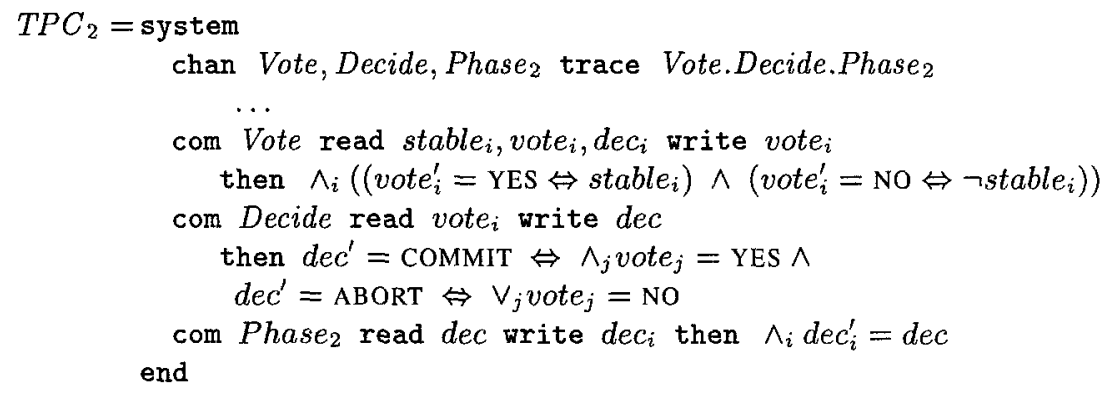

In the next step we make two design decisions. First of all we want to have that the votes are triggered by a request action. Secondly, we want to model that the second phase consist of an information part and an effectuation part. In the first part the participants are informed of the decision, and in the second part some (internal) action is performed to effectuate the decision to be taken. Although this has no visible effect we introduce it to model that in the actual algorithm there is more activity than is specified by the simple input/output specification $A C P$.

The introduction of such internal actions is even simpler than the other two steps: any then relation can be viewed as the composition of the identity relation and itself. The result is a specification with the following trace.

$$
T P C_{3}=\text { system } \ldots \text { trace Req.Vote.Decide.Inform.Eff... end }
$$

In the system above the structure and distribution of processes is not visible as yet. In the next step we want to introduce such information. We decide that all actions but Decide should be split into $n$ different actions, one for every process. This is done in order to allow parallelization later on. The decision should be taken by a single process. Note that this is also a design decision: a different decision would be to split dec again and let all processes decide themselves.

We split all actions into a sequence of $n$ actions, similarly as would be done in ordinary sequential program development in the style of Dijkstra. All conjunctive relations allow to do so. The result is given in figure 7 .

\subsection{Distributing actions}

In the system $T P C_{4}$ there is a lot of superfluous ordering. Many actions are independent, even at a syntactic level. At this stage we exploit these independencies to parallelize the system. In principle all $R e q_{i}$ actions are independent, all Vote ${ }_{i}$ actions are independent, all Inform $_{i}$ actions are independent, and all Effi actions are independent. In fact, all $R e q_{i}$ actions are independent with respect to any other action, but these were introduced to model triggering of the protocol, and therefore we want to have that first the coordinator is triggered (process 1 , using $R e q_{1}$ ) and thereafter the rest is triggered. Obviously this is not a formal interpretation of the specification, but only interpretation of the way the system should function in an environment.

We repeatedly apply the transformation rule PAR to arrive at the following system, where all actions for the coordinator have been grouped into a single sequence as we 


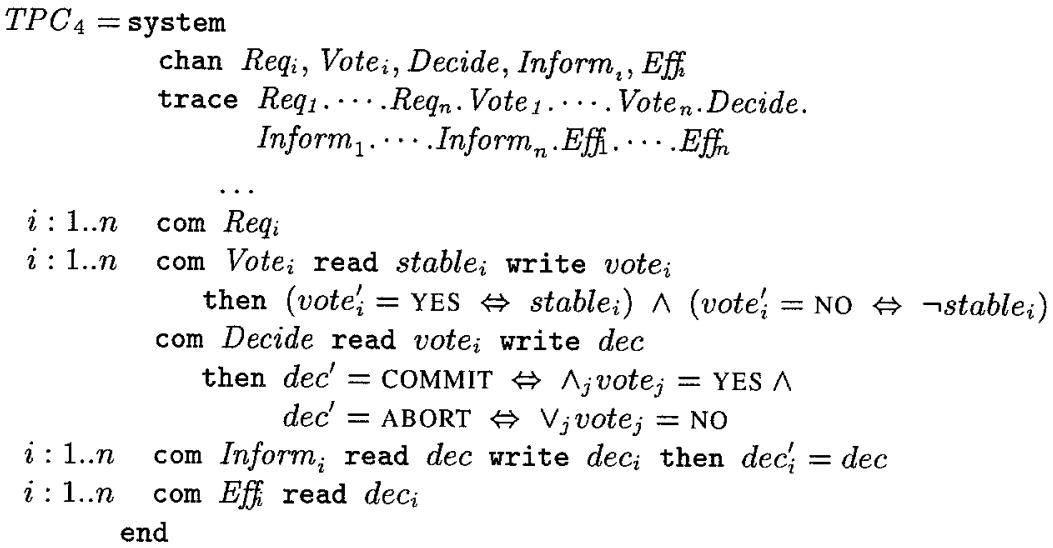

Fig. 7. Structure of $T P C_{4}$

want to arrive at a system consisting of $n$ sequential processes. Thus we do not exploit the full potential of parallelism in the system.

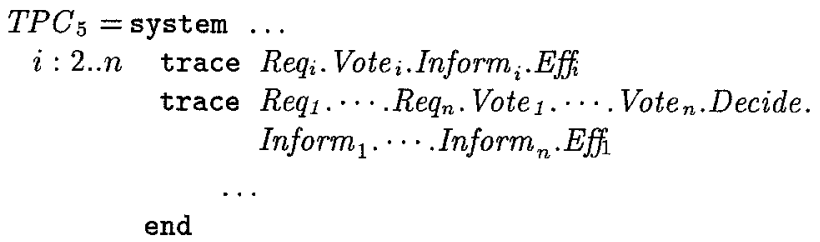

For the above system to be parallelized completely, we have to split the different communication assertions of actions that are used to synchronize components into two parts, one for each of the synchronizing components. For example, a Vote $_{i}$ action is split into two communication assertions, one modeling sending of a value, the other modeling reception of a value. Recall that @ $a$ represents the communicated value. For Vote $_{i}, i \neq 1$ this results in:

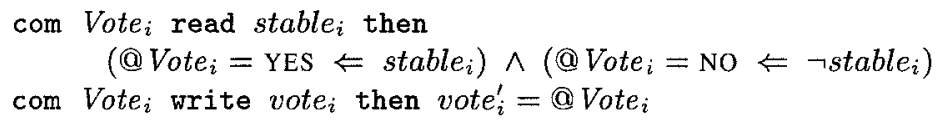

The Decide action and other non-synchronizing actions need not to be split.

\subsection{Final steps}

The system that is the result of the above transformations is now decomposed to the parallel composition of specifications using the SYNC rule, resulting in the structure

$$
T P C_{6}=\operatorname{chan} \alpha \operatorname{SYNC}\left[S_{1}, \ldots, S_{n}\right],
$$


where $\alpha$ is the set of all synchronizing channels and every $S_{i}$ is the specification of a component, $S_{1}$ is the coordinator and $S_{2}$ through $S_{n}$ are the participants. For example, for $2 \leq i \leq n$,

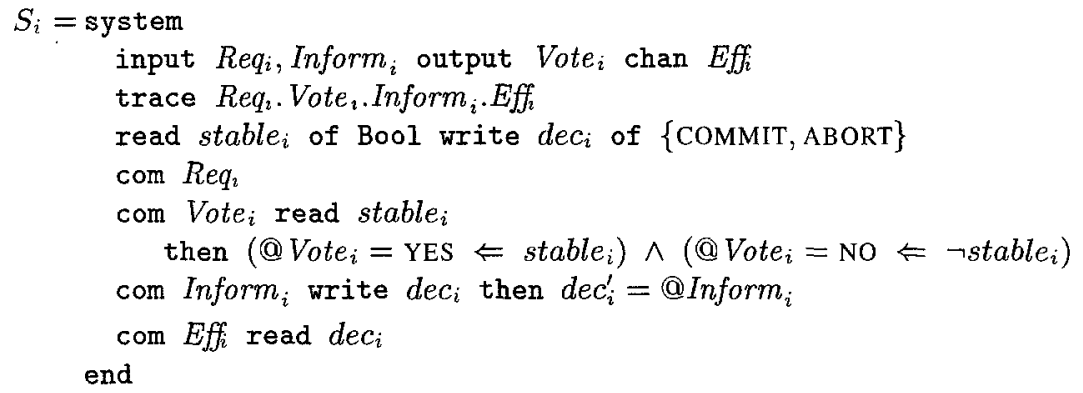

Every specification $S_{i}$ has a single trace assertion only. Since all the communication assertions are implementable, the systems can directly-even automatically-be translated into a sequential implementation [5]. This automatic translation, called Syntax Directed Transformation, results in an OCCAM like implementation, given in figure 8 . In this program we have left $E f f_{i}$ unspecified, but besides that it can directly be in-

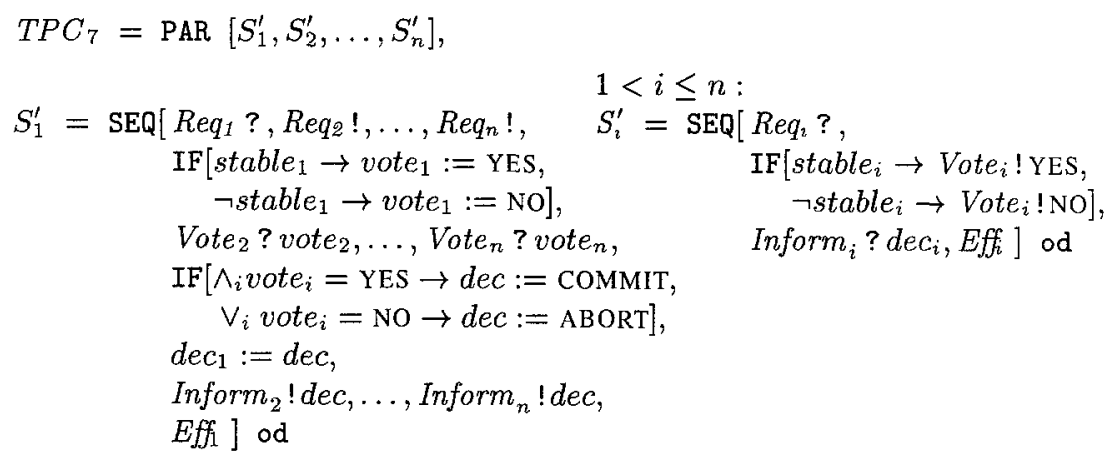

Fig. 8. Centralized Two-Phase Commit

terpreted as an OCCAM program and it is suited for the architecture we had in mind. Concludingly, we have derived an implementation for the atomic commit protocol that satisfies our needs.

\section{The Parallel Approach}

In the parallel strategy one tries to obtain a first solution that is free of any architectural bias. We assume we have a "single step" specification modeling input/output behavior only, such as in the case of $A C P$. The idea is to find a set of actions that, given an initialization and a weakly fair scheduling policy, evolves to a stable state satisfying the 
desired final state [6]. To prove that such an initial solution is indeed a solution to the problem we have to define an invariant $\mathcal{I}$ that is invariant for all actions, which is established by the initialization. Together with the fix point predicate FP of the computation this invariant should establish the postcondition of the specification. In UNITY this fix point is defined as the condition such that no action leads to any changes anymore. In our MIX context this condition changes to the predicate that states that no action is enabled anymore, as only in terminating runs the end state is defined. To show termination we moreover have to specify a metric $\tau$ that decreases under a weakly fair scheduling of the actions, analogously to bound functions in sequential programming. We refer to Chandy and Misra [6] for more details on these ideas.

For this strategy one has a number of proof obligations for the first solution, as was mentioned above. This is due to the fact that there is a rather large "gap" between the initial specification and the first refinement. The larger the distance, the more complicated the proof usually is.

\subsection{An initial solution}

As a first solution we take a set of actions assigning values to the variables vote $_{i}$ and $d e c_{i}$. Initially these variables are assigned the value NONE, so we slightly extend the types of the variables. The solution is given in figure 9. (We continue with $T P C_{8}$ in order to have unique names of specifications.)

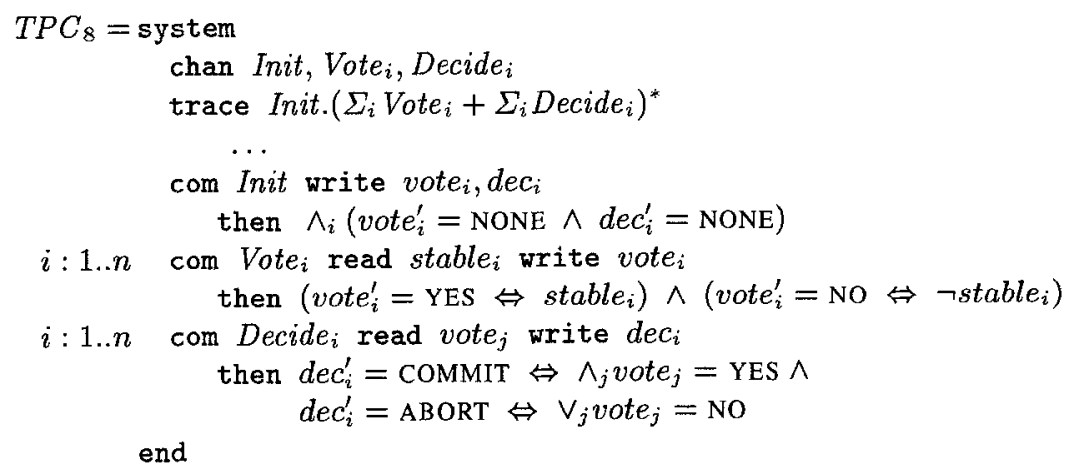

Fig. 9. Structure of $T P C_{8}$

To prove that this program indeed is a refinement of $A C P$ we choose as an invariant $\mathcal{I}$ and have the termination predicate $F P$ :

$$
\begin{gathered}
\mathcal{I}=\wedge_{i}\left(\left(\text { vote }_{i}=\mathrm{YES} \Rightarrow \text { stable }_{i}\right) \wedge\left(\text { vote }_{i}=\mathrm{NO} \Rightarrow \neg \text { stable }_{i}\right) \wedge\right. \\
\left(\text { dec }_{i}=\mathrm{COMMIT} \Rightarrow \wedge_{j} \text { vote }_{j}=\mathrm{YES}\right) \wedge \\
\left.\left(\text { dec }_{i}=\mathrm{ABORT} \Rightarrow \vee_{j} \text { vote }_{j}=\mathrm{NO}\right)\right) \\
F P=\wedge_{i}\left(\text { vote }_{i} \neq \mathrm{NONE} \wedge \text { dec }_{i} \neq \mathrm{NONE}\right) .
\end{gathered}
$$


The bound function is given by the number of variables $v o t e_{i}$ and $\operatorname{dec}_{i}$ that (still) have the value NONE. The initialization trivially ensures $\mathcal{I}$ as no left hand side of any implication holds. Also, all actions may change the value of one variable only, which is changed in the correct way if it changes, preserving $\mathcal{I}$. Finally, given the possible values of the variables and $F P$, we obtain the post condition of $A C P$.

\subsection{Mapping to an architecture}

The above solution is a general solution. We now have to tune it to a certain architecture, that is, $n$ processes with point to point communication. For point to point communication no action may involve variables that are assigned to more than two processors, and may only write a single variable. So we can assign actions to processors, and then have to refine in such a way that the above is indeed satisfied. For a shared variables implementation we would only have to assign actions to processors, without having to both about variables. The variable distribution will be as in the previous protocol, so, for example, the actions Decide ${ }_{i}$ in $T P C_{8}$ are not implementable.

In UNITY one has the concept of superposition that allows to modify actions to a certain extent and to add variables and actions. Here we add an action Decide, that assigns a value to a new, local variable $d e c$. The addition of such an action that simply writes a new variable and does not change the flow of control can always be done.

Besides that superposition we would like to modify the Decide $e_{i}$ actions such that they no longer read the vote $j_{j}$ variables but dec. Otherwise the previous modification would not make much sense. To prove the correctness thereof we have to give a strengthened invariant and $F P$ and prove correctness again.

Finally, the Init action is split in a parallel composition of $n$ actions initializing the variables for every process. The result is a (still unstructured) algorithm that however can be mapped to a point to point architecture.

To map the result of the above steps to a sequential program, we need something extra. We can deduce that every action changes the state exactly once: every $V_{0} e_{i}$ the first time it is executed, Decide as soon as all votes are known or at least one vote is No etcetera. All other executions of the actions do not change the state. Based on this, we can define a bijective mapping $s:\left|A_{i}\right| \rightarrow A_{i}$ that defines the order in which the set of actions $A_{i}$ for process $i$ is executed. This mapping should be such that the state after the $j$-th action ensures that $s(j+1)$ is enabled and changes the state. In our case, the mapping (obviously) corresponds to the order in which the actions are executed in $T P C_{4}$, modulo some renaming. The rule to introduce such ordering is given in [4], we here restrict ourselves to showing the result. Finally, we have to add actions $E f f_{i}$ as well, which can be done without any problems in this case, as these are local actions that do not change the state. The result is given in figure 10 . If we rename the actions Init $t_{i}$ into $R e q_{i}$ and Decide $_{i}$ into Inform $_{i}$ the rest of the derivation is the same as in section 5, but for the implementation of $R e q_{i}$ which now does change the state. This concludes the sketch of the derivation. 


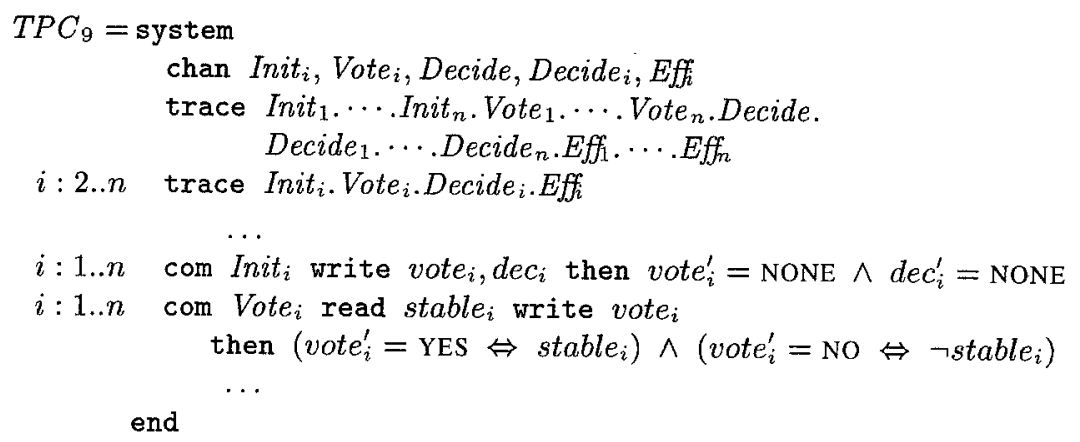

Fig. 10. Structure of $T P C_{9}$

\section{Using the Architectural Strategy}

The two previous sections used strategies that delay architectural decisions to as late stage as possible. In the architectural approach we do the opposite: the synchronization skeleton of the system one is aiming at is introduced as a first step, and only thereafter state transitions are analyzed. This can have the advantage that certain simplifications due to the architecture can be exploited already at an early stage. Here we give a sketch of how to apply this strategy to $A C P$.

Following the architectural strategy as described in section 2 we first introduce new local channels Req Rote $_{i}$, Decide, Inform $_{i}$ and Effi and define in what way the different communications synchronize, the so-called synchronization skeleton. This introduction doesn't change the specified state transitions. Only the ordering of the new channels is constraint by integrating them into the trace assertions. Such introduction is therefore allowed as long as the language generated projected on $A C P$ doesn' t change. The result is given in figure 11 .

The next step in this strategy is the extension of the state space as required. Here we introduce the local variable dec and give the $A C P$ action the right to write into it. For the third part of the strategy we need a rule that allows to shift state transitions over actions. Informally this rule states that, if an action $c$ is always followed by an action $d$, then we can move part of the effect of $c$ to $d$, provided certain restrictions on variables accesses are fulfilled. To apply this rule we must partition the set of actions into three sets $C h_{1}, C h_{2}$ and $\{c, d\}$, where $d$ is the channel we want to move part of $c$ to, $C h_{1}$ is the set of channels that might interfere with $c$ or $d$, and $C h_{2}$ all other channels. The trace language should be such that we can add the trace assertion trace $\left(C h_{1}^{*} . c . d\right)^{*}$ without changing the language. Usually we can take $C h_{1}$ to be the set of actions we can ensure to be ordered to the left or to the right of both $c$ and $d$. This set should then contain all interfering actions for $c$ and $d$. The rule is given in figure 12, where $P ;{ }_{\vec{y}} Q$ is the relational composition of predicates $P$ and $Q$, defined as

$$
P ;{ }_{\bar{y}} Q \stackrel{\text { def }}{=} \exists \bar{y}^{\prime \prime} . P\left[\bar{y}^{\prime \prime} / \bar{y}^{\prime}\right] \wedge Q\left[\bar{y}^{\prime \prime} / \bar{y}\right] .
$$




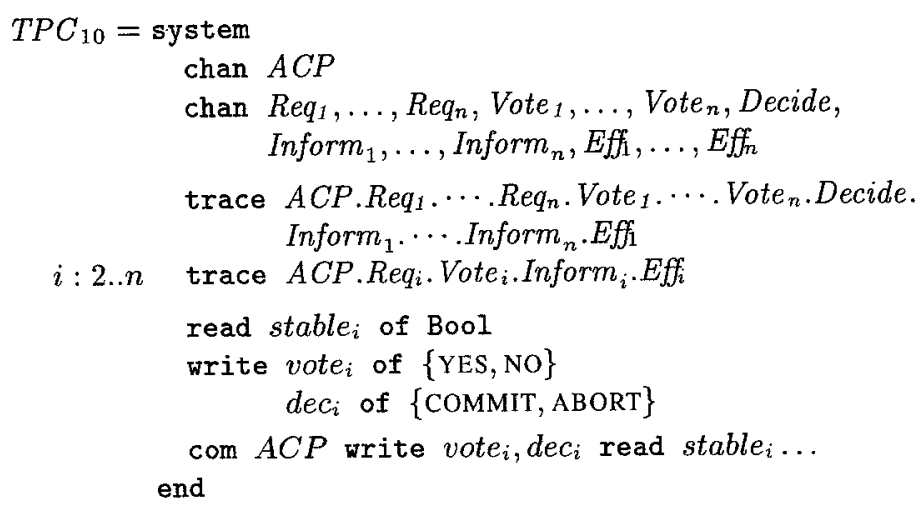

Fig. 11. After adding the synchronization skeleton, $T P C_{10}$

In this case applying the SHIFT rule is rather simple, as there is only a limited amount

\begin{tabular}{|c|c|}
\hline \multicolumn{2}{|c|}{ SHIFT: Shift effects } \\
\hline $\begin{array}{l}\text { system } \triangle T A C A l V l C \\
\text { chan } c, d \text { trace }\left(C h_{1}^{*}, c . d\right)^{*} \\
\text { com } c \text { write } \bar{w} \text { read } \bar{r} \\
\text { when why then } P ;{ }_{\bar{w}} Q \\
\text { com } d \text { write } \bar{w} \text { read } \bar{r} \\
\text { when wh then } R \\
\text { end }\end{array}$ & $\begin{array}{l}\text { system } \Delta T A C A l V l C \\
\text { chan } c, d \text { trace }\left(C h_{1}^{*} . c . d\right)^{*} \\
\text { com } c \text { write } \bar{w} \text { read } \bar{r} \\
\text { when } w h_{c} \text { then } P \\
\text { com } d \text { write } \bar{w} \text { read } \bar{r} \\
\text { when } w h_{d} \text { then }\left(Q ;{ }_{\bar{w}} R\right) \\
\text { end }\end{array}$ \\
\hline $\begin{array}{l}\text { channels } \\
\operatorname{Vars}(\mathrm{Cl}\end{array}$ & $\begin{array}{l}\text { oned into } C h_{1}, C h_{2} \text { and }\{c, d\} \\
A s\left(C h_{2}\right) \backslash \bar{w} \text { and free }\left(w h_{d}\right) \cap \bar{u}\end{array}$ \\
\hline
\end{tabular}

Fig. 12. Shifting effects

of parallelism in the algorithm due to the sequential structure of the coordinator. So we iteratively rewrite the then predicate belonging to $A C P$ to the composition of two predicates. Of course this is not possible in general: we should carefully select the order in which to apply the SHIFT rule. For example, take the action Inform $_{i}$. One can rewrite the predicate corresponding to $A C P$, that is, the full specification, as

$$
P ;{ }_{\text {dec }} \wedge_{i}\left(\operatorname{dec}_{i}^{\prime}=\operatorname{dec}\right) \text {. }
$$

Moreover, this conjunction can be rewritten as

$$
d e c_{1}^{\prime}=\operatorname{dec} ; \emptyset d e c_{2}^{\prime}=\operatorname{dec} ; \emptyset \ldots ; \emptyset \operatorname{dec}_{n}^{\prime}=\operatorname{dec},
$$

as " ; $\emptyset$ " simply is conjunction due to the definition of " ; $\bar{y}$." Therefore we can move the predicate $d e c_{i}^{\prime}=\operatorname{dec}$ to its place, starting with $i=n$ using SHIFT with the channel set 
$C h_{1}=\left\{\right.$ Inform $_{i+1}, \ldots$, Inform $\left._{n}\right\}$ and the channel set $C h_{2}$ is everything but $A C P$ and $\left\{\right.$ Inform $_{i}, \ldots$, Inform $\left._{n}\right\}$, and iteratively removing the whole of the conjunction. As only the channels Inform $_{i}$ and $A C P$ access any variables the side conditions for the SHIFT rule are obviously fulfilled. Thereafter we can shift making the decision to Decide. The part for the Vote actions is then analogous to the Inform part. Finally, we arrive at an algorithm that is almost the same as $T P C_{5}$, but for the fact that all traces start with $A C P$. As $A C P$ now has become a local channel with empty effect and is always enabled, we can remove it from the traces without changing the effect of the system as a whole. The result is exactly $T P C_{5}$ and we can proceed as in section 5.

\section{Implementations for alternative architectures}

Having developed a first implementation of the atomic commit problem for a certain architecture in different ways, it is an interesting question to see how this helps when developing solutions for other architectures. It would be nice if one could reuse parts of the proof due to overlapping development strategies. In doing so one also gets insight in the design decisions that are taken during the development and how they relate to the eventual implementations.

In [4] we derived different versions of the Two-Phase Commit protocol, such as Linear TPC for chain networks, a Tree based version, assuming a tree network, a solution for a Ring network, and finally a Decentralized TPC for fully connected networks. For the latter also an optimized version was derived.

In figure 13 we have sketched how the different transformation steps of the protocols relate. Arrows denote transformation steps, and dotted regions denote sets of highly related algorithms, in the sense that the transformation steps going from one region to another are completely analogous. All derivations were performed using the layered strategy. Each number in the graph correspond to the numbers of the specifications in

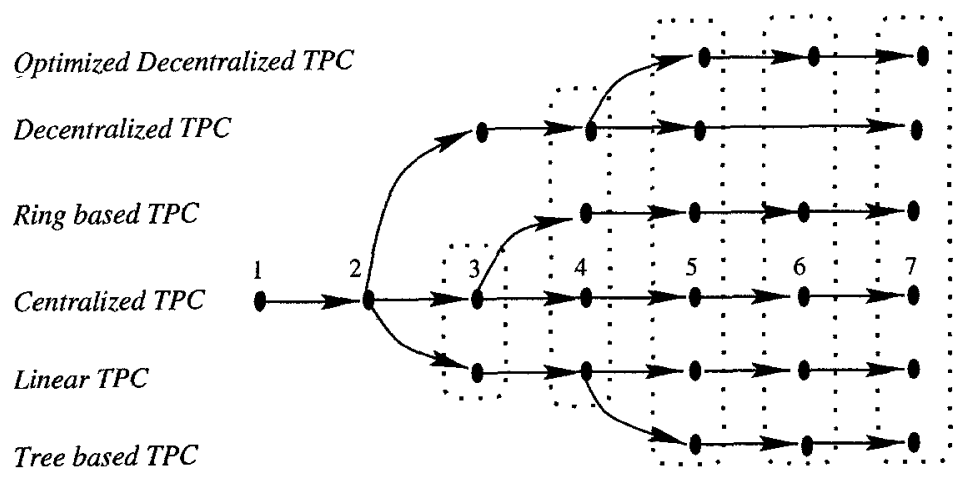

Fig. 13. Relations between different derivations

this paper. Many derivations share large parts of their paths, either almost literally, or by 
analogy in steps. The extra derivations were thus even easier than the derivations given in the previous sections.

\section{Conclusion}

In this paper we studied and compared three different strategies for transformational design of parallel systems: the layered strategy, the parallel strategy, and the architectural strategy. All three strategies were employed within a single formalism, resulting in a comparison of strategies on a non-trivial example. In this example it has been shown that the use of strategies helps in designing systems and designing variants of systems. Proofs of alternative implementations were lead by the first proof, and different proofs could reuse large parts of each other. The similarities in these proofs were induced by the strategy.

Also the derivation of optimizations is supported by the use of strategies. Optimizations can also be seen as variants, often variants that differ to a rather small extent. It is however not yet clear whether we have the right set of rules for such applications.

Having a single formal basis for all strategies enables the integration within existing or future tools, such as the tools supporting MIX transformations that are being built at the University of Oldenburg. The idea is to add strategies as meta-level transformations with user interaction to guided developers through the long and complicated design trajectory as smoothly as possible.

A question to be answered in the near future is how to formalize strategies. For theorem provers strategies can either be formalized on a meta-level or using the underlying logic, as has been studied in [5]. In the context of automatic tool synthesis there is interesting work by Steffen and Margaria [22] using a modal (dynamic) logic to specify similar constraints.

Another interesting topic is the introduction of asynchronous message passing. Some work in this direction has already been done by Fischer [10]. This would also allow to model things like duplication, loss, or corruption of messages. It is not yet clear how this would influence the approach taken here. For a certain class of systems it seems to be possible to define a strategy to transform synchronous systems to asynchronous systems. Furthermore, the whole MIX setup does not allow refinement of interfaces. One can only relate systems that have the same interface but possibly different behavior. This lead to the restriction that all channels are local channels in this report. The introduction of interface refinement, in the style of Gerth and Kuiper [11] or Stomp and Siegel [24].

Acknowledgments. The authors would like to thank Stephan Rössig for comments in early stages of this work, and Stephan Kleuker for rigorously reading the full report. The term "architectural strategy" was suggested by Anders Ravn. Finally, we would like to thank Ernst-Rüdiger Olderog for useful suggestions.

\section{References}

1. R. Back. Refinement calculus, Part II: Parallel and Reactive Programs. In de Bakker, de Roever, and Rozenberg, editors, Stepwise Refinement of Distributed Systems, LNCS 430, pages 67-93. Springer-Verlag, 1990. 
2. R. Back and K. Sere. Stepwise refinement of action systems. Structured Programming, 12:17-30, 1991.

3. P. Bernstein, V. Hadzilacos, and N. Goodman. Concurrency Control and Recovery in Database Systems. Addison-Wesley, 1987.

4. J. Bohn and W. Janssen. From a single specification to many implementations - many roads lead to parallelism. Technical report, University of Oldenburg, 1995. Available at ftp://ftp.informatik. uni-oldenburg.de/pub/procos/.

5. J. Bohn and S. Rössig. On automatic and interactive design of communicating systems. In E. Brinksma, W. Cleaveland, K.G. Larsen, T. Margaria, and B. Steffen, editors, Proceedings of the First TACAS workshop, LNCS 1019, pages 216-247. Springer-Verlag, 1995.

6. R. Chandy and J. Misra. Parallel Program Design: A Foundation. Addison-Wesley, 1988.

7. C. Chou and E. Gafni. Understanding and verifying distributed algorithms using stratified decomposition. In Proceeding 7th ACM Symposium on Principles of Distributed Computing, 1988.

8. T. Elrad and N. Francez. Decomposition of distributed programs into communication closed layers. Science of Computer Programming, 2:155-173, 1982.

9. J. P. Bowen et al. A ProCoS II project description: ESPRIT Basic Research project 7071. Bulletin of the EATCS, 50:128-137, 1993.

10. C. Fischer. Transformation von synchronen SL-Specifikationen von Telekommunikationssytemen in asynchrone SL-Specifikationen. Master's thesis, University of Oldenburg, 1995. In German.

11. R. Gerth, R. Kuiper, and J. Segers. Interface refinement in reactive systems. In Proceedings CONCUR' '92, LNCS 630, pages 77-94. Springer-Verlag, 1992.

12. C.A.R. Hoare. Communicating Sequential Processes. Prentice-Hall, 1985.

13. W. Janssen. Layered Design of Parallel Systems. PhD thesis, University of Twente, 1994.

14. W. Janssen, M. Poel, and J. Zwiers. Action systems and action refinement in the development of parallel systems. In Proceedings of CONCUR '91, LNCS 527, pages 298-316. Springer-Verlag, 1991.

15. L. Lamport. The Temporal Logic of Actions. ACM TOPLAS, 16(3):872-923, 1994.

16. C. Lengauer. Loop parallelization in the polytope model (invited talk). In Eike Best, editor, Proceedings CONCUR '93, LNCS 715, pages 398-416. Springer-Verlag, 1993.

17. E.-R. Olderog. Towards a design calculus for communicating programs (invited paper). In Proceedings of CONCUR '91, LNCS 527, pages 61-77. Springer-Verlag, 1991.

18. E.-R. Olderog and S. Rössig. A case study in transformational design of concurrent systems. In M.-C. Gaudel and J.-P. Jouannaud, editors, TAPSOFT '93, LNCS 668, pages 90-104. Springer-Verlag, 1993.

19. E.-R. Olderog, S. Rössig, J. Sander, and M. Schenke. ProCoS at Oldenburg: The interface between specification language and occam-like programming language. Berichte aus dem Fachbereich Informatik 3, University of Oldenburg, 1992.

20. S. Rössig. A Transformational Approach to the Design of Communicating Systems. PhD thesis, University of Oldenburg, 1994.

21. J. Spivey. The Z Notation: A Reference Manual. Prentice Hall, 1989.

22. B. Steffen, T. Margaria, and A. Claßen. Heterogeneous analysis and verification for distributed systems. Technical Report MIP-9509, University of Passau, 1995.

23. F. Stomp. A derivation of a broadcasting protocol using sequentially phased reasoning (extended abstract). In L. Logrippo, R. Probert, and H. Ural, editors, Proceedings 10th IFIP symp. on Protocol Specification, Testing and Verification, pages 19-32. Elsevier Science Publishers, 1990.

24. F. Stomp and M. Siegel. Extending the limits of sequentially phased reasoning. In P. Thiagarajan, editor, Proceedings FST \& TCS 14, LNCS 880. Springer-Verlag, 1994. 